\title{
Numerical Analysis on Variation of Dynamic Response of Girder Bridges with Torsional Reinforcement Panels
}

\author{
Jae-Yoon Kang ${ }^{1, a}$, Jong-Won Kwark ${ }^{1}$, Sung-Yong Park ${ }^{1}$ and K.T. Kim² \\ ${ }^{1}$ Korea Institute of Civil Engineering and Building Technology, Structural Engineering Research Institute, Goyang, 411-712 Korea \\ ${ }^{2}$ BNG Consultants, Sungnam, 463-825 Korea
}

\begin{abstract}
The dynamic flexural behaviour of the railway bridge is influenced by its torsional behaviour. Especially, in the case of girder railway bridges, the dynamic response tends to amplify when the natural frequency in flexure $\left(1^{\text {st }}\right.$ vibration mode) is close to that in torsion ( $2^{\text {nd }}$ vibration mode). In order to prevent such situation, it is necessary to adopt a flexural-to-torsional natural frequency ratio larger than $120 \%$. This study proposes a solution shifting the natural frequency in torsion to high frequency range and restraining torsion by installing concrete panels on the bottom flange of the girder so as to prevent the superposition of the responses in the girder bridge. The applicability of this solution is examined by finite element analysis of the shift of the torsional natural frequency and change in the dynamic response according to the installation of the concrete panels. The analytical results for a $30 \mathrm{~m}$-span girder railway bridge indicate that installing the concrete panels increases the natural frequency in torsion by restraining the torsional behaviour and reduces also the overall dynamic response. It is seen that the installation of $100 \mathrm{~mm}$-thick concrete panels along a section of $4 \mathrm{~m}$ at both extremities of the girder can reduce the dynamic response by more than $30 \%$.
\end{abstract}

\section{Backgrounds}

In railway bridges, the girder bridge is vulnerable to torsion owing to its open section. In double-track bridges, the passage of a train in one track acts as an eccentric load in the whole cross-section, which makes the torsional behaviour have significant influence on the dynamic response. The dynamic response of the railway bridge appears through the coupling of its flexural and torsional behaviours. The first vibrational mode, which has generally the largest contribution, exhibits flexural behaviour, and the $2^{\text {nd }}$ mode shows torsional behaviour. Superposition of the dynamic response occurs when the natural frequencies of these two modes become closer, resulting in the amplification of the dynamic response of the whole structural system under the passage of a train. In order to minimize the effect caused by the superposition of these vibrational modes, a difference in the natural frequency between the $1^{\text {st }}$ and $2^{\text {nd }}$ modes larger than $20 \%$ should be adopted.

By experience, it is known that, in ballasted tracks, the damping effect brought by the mass of the gravel layer results in a natural frequency of the torsional vibration mode ( $2^{\text {nd }}$ mode) higher by more than 1.25 times that of the bending mode ( $1^{\text {st }}$ mode), that is a difference larger than $25 \%$. However, this difference is likely to be smaller than $20 \%$ in concrete track bridges, which means high risk for the amplification of the response to occur due to the mode superposition.
Accordingly, this study proposes a solution increasing the torsional rigidity of the structural system to improve the dynamic behaviour and prevent the torsional behaviour known to be the weakness of the girder-type railway bridge. Specifically, this solution modifies the structural system by installing articulated reinforcement panels connecting the bottom flanges of the girder so as to control the torsional behaviour without affecting the flexural behaviour. The effects of this solution are examined by finite element analysis with regard to the change in the natural frequencies and dynamic response.

\section{Alternatives for the Control of Torsional Behaviour}

As a torsional reinforcement solution improving the dynamics of the girder-type railway bridge, the increase of the torsional rigidity by connecting laterally the bottom flanges of the girder was proposed. Basically, one alternative is to dispose concrete panels with constant thickness and width on the bottom flanges (Figure 1(a)), and another alternative is to arrange steel bars or tubes with definite stiffness at regular intervals between the bottom flanges (Figure 1(b)). The first alternative using the concrete panels was finally selected considering the workability and practicability on site as well as the effect of the reinforcement system and the constructability in railway bridges in service. Accordingly, the torsional reinforcement effect of this solution is evaluated.

\footnotetext{
${ }^{\mathrm{a}}$ Corresponding author: jykang@kict.re.kr
} 


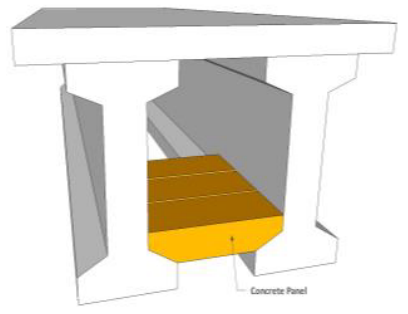

(a) Reinforcement by concrete panels.

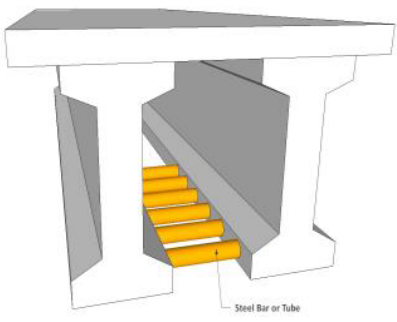

(b) Reinforcement by steel bars or tubes.
Figure 1. Solutions for torsional reinforcement of girder bridge.

\section{Dynamic Analysis for the Evaluation of Torsional Reinforcement Effect}

\subsection{Summary of dynamic analysis}

The $30 \mathrm{~m}$-span girder railway bridge with double track and concrete ballast shown in Figure 2 is considered for evaluating the applicability of the torsional reinforcement system, that is to examine the change in the natural frequency according to the dimensions of the concrete panels and the condition of their installation. The width of the panels is fixed to $50 \mathrm{~cm}$ and, the optimal thickness and position of the panels are determined so as to obtain the torsional reinforcement effect by taking the thickness and position of the panels inside the bridge span as variables. In the analysis, the thickness of the panels is increased by step of $10 \mathrm{~mm}$ from $20 \mathrm{~mm}$ to $200 \mathrm{~mm}$, and three dispositions of the panels are considered: over the whole span, over sections of $10 \mathrm{~m}$ and $5 \mathrm{~m}$ at both extremities of the bridge.

Figure 3 illustrates the three-dimensional analysis model applying appropriate constraints and finite elements including beam elements, shell elements, solid elements and spring elements to reflect the characteristics of the girder bridge. The track structure is also modelled for accurate analysis. The girders and cross-beams are modelled by 2 node beam elements and the deck by 4-node shell elements. Beam-type constraint is applied for the nodal connection between the girder and bearing and the girder-deck connection considering the depth of the girder. Table 1 arranges the modelling method for each structural element.

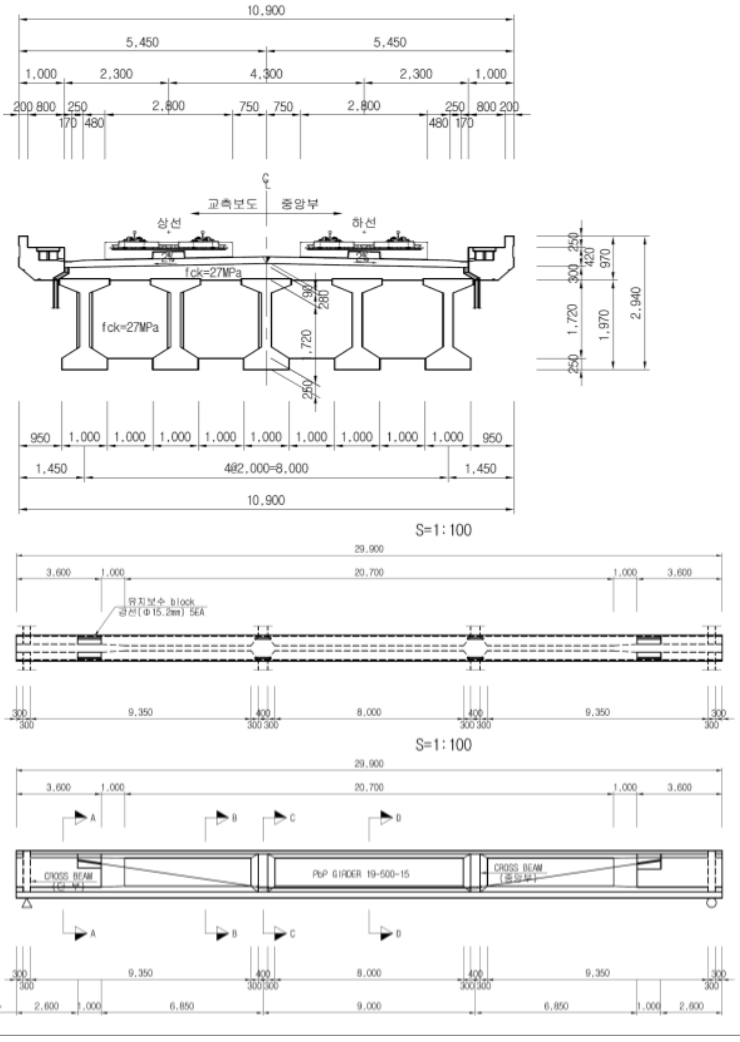

Figure 2. Cross-sectional views of the bridge $(30 \mathrm{~m}$, double track, concrete track).

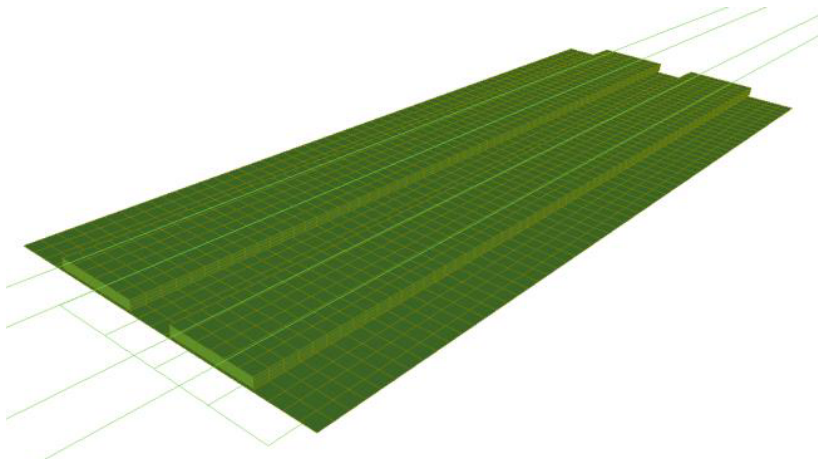

Figure 3. Dynamic analysis model.

Table 1. Modelling method of concrete track bridge.

\begin{tabular}{|c|c|c|c|}
\hline Structure & Structural component & Element/constraint & Remarks \\
\hline \multirow{4}{*}{$\begin{array}{c}\text { Bridge } \\
\text { structure }\end{array}$} & Girder & 2-node beam & \\
\cline { 2 - 4 } & Cross-beam & 2-node beam & Including PCL mass effect \\
\cline { 2 - 4 } & Girder-deck connection & 4-node shell & \\
\cline { 2 - 4 } & Girder-bearing nodal link & Beam-type constraint & \\
\cline { 2 - 4 } & Parapet concrete & Stiffness ignored, mass considered & \\
\hline \multirow{4}{*}{$\begin{array}{c}\text { Concrete } \\
\text { track }\end{array}$} & PCL & Stiffness ignored, mass considered & \\
\cline { 2 - 4 } & TCL (including sleeper) & 8-node solid & Modelled as 3 layers (total depth 360 mm) \\
\cline { 2 - 4 } & TCL bottom node-deck connection & Node-to-surface constraint & \\
\cline { 2 - 4 } & Rail & 2-node beam & \\
\cline { 2 - 4 } & Sleeper & Ignored & \\
\cline { 2 - 4 } & Sleeper node-TCL upper surface & Node-to-surface constraint & \\
\cline { 2 - 4 } & Outer rail-ground & Grounded spring & \\
\cline { 2 - 4 } & & & \\
\hline
\end{tabular}




\subsection{Eigenvalue analysis of non-reinforced bridge}

Prior to the analysis of the disposition of the torsional reinforcement panels, eigenvalue analysis of the nonreinforced bridge is performed and the difference ratio in the natural frequency between the flexural and torsional modes is examined.

The results are arranged in Table 2. For the considered concrete track girder bridge, the natural frequency ratio of the $1^{\text {st }}$ mode to the $2^{\text {nd }}$ mode is 1.14 . This indicates that, for the example bridge, the dynamic behaviour must be improved by increasing the ratio to a value larger than 1.2 by means of torsional reinforcement.

Table 2. Comparison of natural frequencies (before application of reinforcement panels).

\begin{tabular}{|c|c|c|c|}
\hline Mode & $\begin{array}{c}\text { Natural } \\
\text { frequency } \\
(\mathrm{Hz})\end{array}$ & Ratio $\boldsymbol{f}_{\boldsymbol{n}} \boldsymbol{f}_{\mathbf{1}}$ & Mode shape \\
\hline $1^{\text {st }}\left(f_{1}\right)$ & 4.765 & - & \\
\hline $2^{\text {nd }}\left(f_{2}\right)$ & 5.433 & $\mathbf{1 . 1 4}$ & \\
\hline $3^{\text {rd }}\left(f_{3}\right)$ & 10.401 & 2.18 & \\
\hline $4^{\text {th }}\left(f_{4}\right)$ & 16.193 & 3.40 & \\
\hline
\end{tabular}

\subsection{Analysis of disposition of torsional reinforcement panels}

The analytical results for the evaluation of the effective arrangement section of the torsional reinforcement panels within the bridge span are shown in Figure 4.

In Figure 4, the horizontal coordinate is the volume of concrete panels obtained by converting the thickness change into volume. Since it appears that identical natural frequency ratios are achieved for the case arranging panels all over the bridge span and the case arranging panels at both ends over about $2 / 3$ of the girder span, it can be verified indirectly that installing panels at the centre of the girder has no effect on the torsional reinforcement nor on the dispersion of the natural frequencies.

Moreover, the comparison of the case arranging the panels over $10 \mathrm{~m}$-long sections at the ends and that arranging the panels over $5 \mathrm{~m}$-long sections at the ends reveals that the latter provides relatively large dispersion of the natural frequencies even with smaller volume of concrete.

In view of the thickness of the panels, it is seen that larger thickness increases the scaling up of the natural frequency. For the arrangement of panels over $5 \mathrm{~m}$-long sections at the ends, thickness larger than $40 \mathrm{~mm}$ can amplify the natural frequency by more than $20 \%$. In other words, the localized arrangement of the torsional reinforcement panels at both ends of the bridge span appears to improve the torsional resistance owing to the closure of the cross-section.

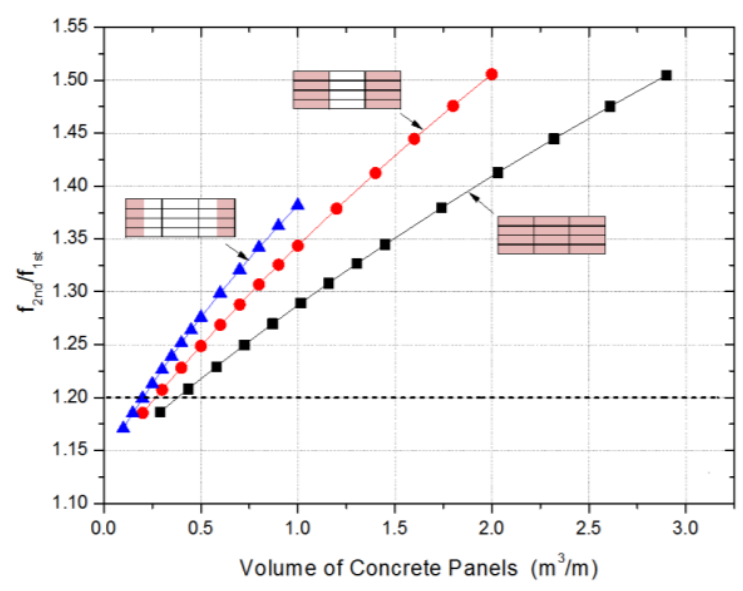

Figure 4. Comparison of natural frequency ratio with respect to disposition of torsional reinforcement panels.

\subsection{Moving load analysis for the evaluation of the improvement of dynamic response}

Moving load analysis is conducted for the case arranging the reinforcement panels over $4 \mathrm{~m}$-long sections at the ends to compare the improvement of the dynamic behaviour brought by the torsional reinforcement panels. Comparison is done with the moving load analysis results relative to the non-reinforced girder. The considered moving loads are the Korean KTX train, the Saemaeul train, the cargo train and the HEMU train currently under development. The analysis results are arranged in Figures 5 to 7.

In Figure 5, the peak acceleration at low speed exhibits slightly large value close by about $1 \%$ to that at resonance speed when the reinforcing panels are installed. However, the value in the non-reinforced case is seen to be very similar to that in the reinforced case. At speed higher than $250 \mathrm{~km} / \mathrm{h}$, the acceleration response tends to reduce owing to the presence of the reinforcement panels. Concretely, the peak acceleration experiences a reduction by $11.5 \%$ and $3.4 \%$ under the respective passage of the KTX train and HEMU train through the installation of the reinforcing panels.

The effect of the torsional reinforcement panels can be clearly identified in view of the vertical displacement and twist responses in Figure 6 and Figure 7, respectively. Figure 6, which plots the difference in the maximum vertical displacement per speed according to the installation of the reinforcing panels, shows that the peak response reduces by approximately $3 \%$ to $8 \%$.

In Figure 7, the twist response is seen to decrease by more than $30 \%$ owing to the installation of the torsional reinforcement panels. The restraint of the torsional behaviour by the reinforcing panels seems thus to have largest reduction effect on the twist response. 


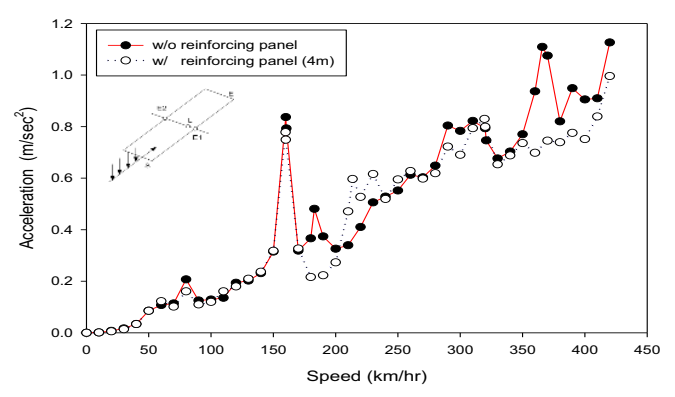

(a) KTX train

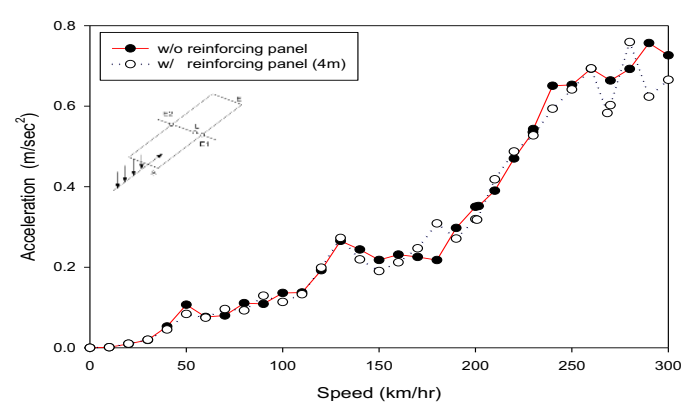

(c) Saemaeul train

Figure 5. Comparison of peak acceleration response.

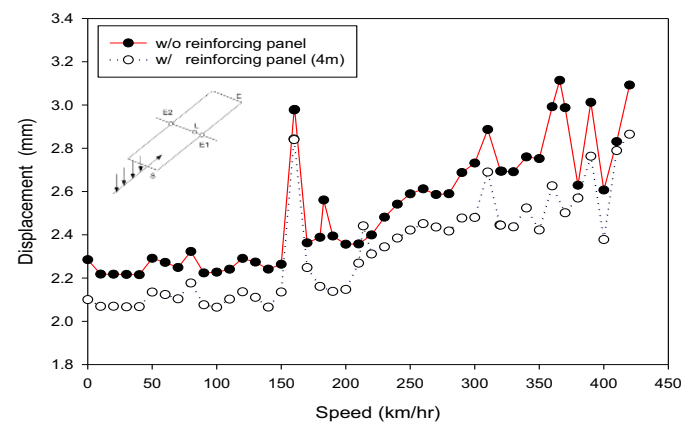

(a) KTX train

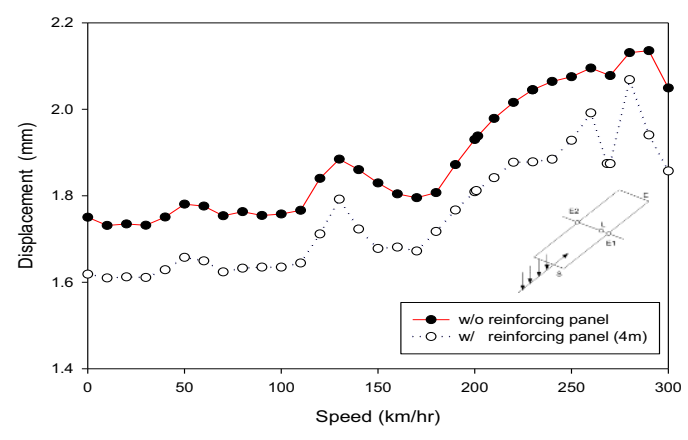

(c) Saemaeul train

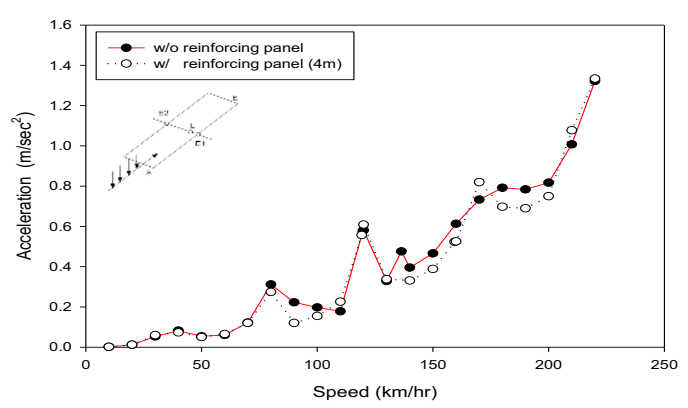

(b) Cargo train

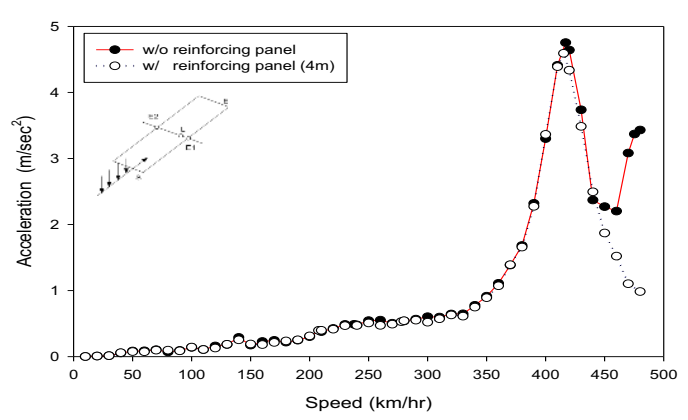

(d) HEMU train (12 coaches)

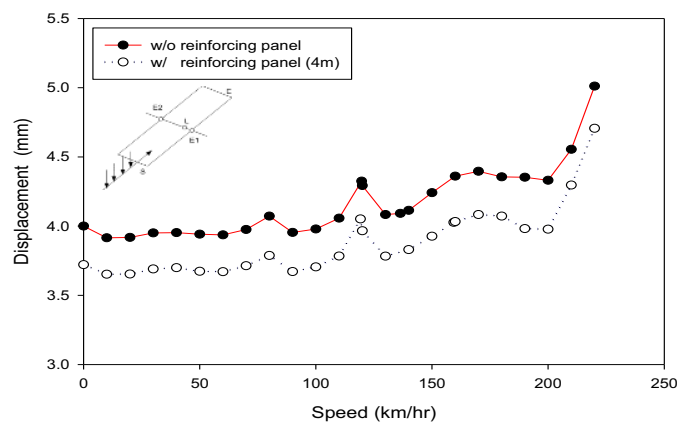

(b) Cargo train

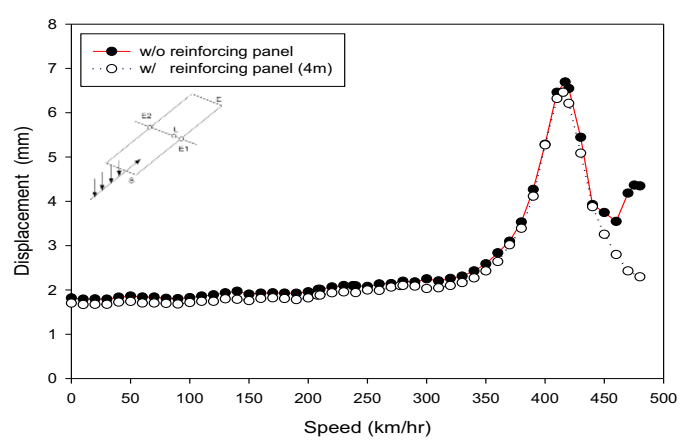

(d) HEMU train (12 coaches)

Figure 6. Comparison of vertical displacement response. 


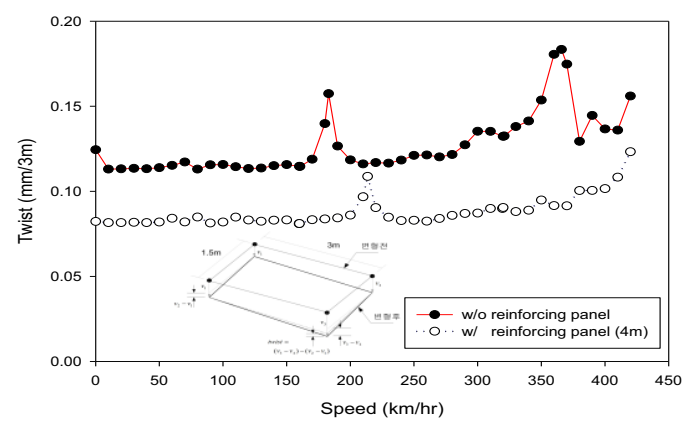

(a) KTX train

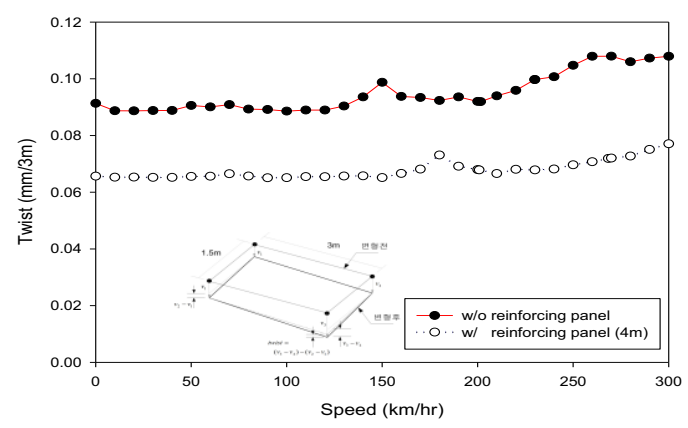

(c) Saemaeul train

Figure 7. Comparison of twist response.

\section{Conclusions}

This paper proposed the installation of concrete reinforcement panels in girder-type railway bridge as a solution supplementing its vulnerability to torsion by increasing the torsional rigidity. The lateral connection of the girders by the torsional reinforcement panels intends to amplify the natural frequency of the flexural vibration mode and suppress the effect of the superposition of flexural and torsional behaviours which, in turn, will result in the improvement of the dynamic stability of the structural system.

Dynamic analysis was performed to examine the enlargement of the difference between the first and second modes by the installation of the torsional reinforcing panels and verify the improvement of the dynamic behaviour of the railway bridge. To that goal, the increase of the natural frequency in torsion of a $30 \mathrm{~m}$ span concrete track girder-type railway bridge was investigated with respect to the thickness and arrangement of the torsional reinforcement panels. In view of the results, the natural frequency in torsion of the girder bridge increased according to the installation of the reinforcing panels, which enlarged the difference with the natural frequency in flexure and minimized the effect caused by the superposition of the two modes.

Moving load analysis was also conducted using the KTX train, cargo train, Saemaeul train and HEMU train (12 coaches) in order to verify the actual improvement of the dynamic behaviour produced by the installation of the torsional reinforcement panels. The results confirmed the restraint of torsion by the torsional reinforcement panels through the reduction of twist response by more than

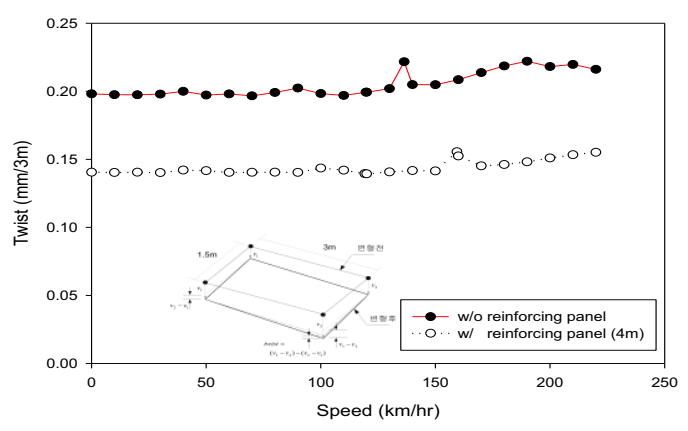

(b) Cargo train

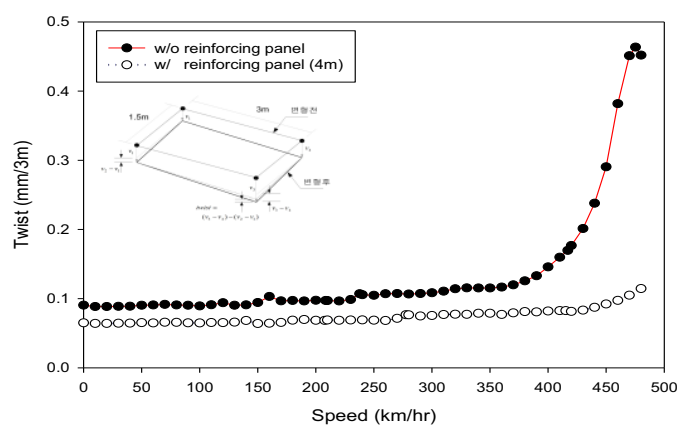

(d) HEMU train (12 coaches)

$30 \%$. Besides, the maximum values of the vertical displacement and vertical acceleration responses were also seen to reduce by approximately $5 \%$ on the average. Overall reduction of the response could be observed within the speed range of each type of train. 